\title{
Comparison of strategies and goals for treatment of chronic constipation among gastroenterologists and general practitioners
}

\author{
Dan Carter ${ }^{\mathrm{a}, \mathrm{c}}$, Eytan Bardan ${ }^{\mathrm{a}, \mathrm{c}}$, Ram Dickman ${ }^{\mathrm{b}, \mathrm{c}}$ \\ Chaim Sheba Medical Center, Tel Hashomer; Rabin Medical Center, Petach Tikva; Tel Aviv University, Israel
}

\begin{abstract}
Background Although guidelines have been published for the treatment of chronic constipation, little is known about the actual treatment strategies, the definitions of drug efficacy, the parameters for drug selection, and the conceived limitations of the available treatments. The purpose of this study was to address these issues by comparing treatment strategies among gastroenterology specialists (GIs) and general practitioners (GPs).
\end{abstract}

Methods An internet survey was sent nationwide and at random to GIs and GPs in order to define treatment strategies, drug efficacy, main parameters for drug selections and the main limitations of the available drug therapy.

Results Forty GIs and 132 GPs answered the survey. The maximal sample error was $\pm 13.4 \%$ and $\pm 8.8 \%$. Treatment strategies varied considerably between GPs and GIs. The major parameters for drug selection were related to drug safety among GIs and to clinical outcome among GPs. The conceived limitations of drug therapy included lack of experience and unwanted side effects.

Conclusions Awareness of the possible treatment options and the recommended order of prescription differs between GIs and GPs. There are still unmet needs for optimizing the treatment for chronic constipation.

Keywords Constipation, treatment, laxatives, survey

Ann Gastroenterol 2017; 31 (1): 1-6

\section{Introduction}

Chronic constipation (CC) is a common complaint that affects up to $15 \%$ of the general western population [1-3]. The symptoms consistent with CC are variable, including straining, hard stools, a sensation of incomplete evacuation, a sense of anorectal obstruction, manual maneuvers to facilitate defecation, and $<3$ spontaneous bowel movements per week [4]. CC causes significant reduction in the quality

Department of a Gastroenterology, Chaim Sheba Medical Center, Tel Hashomer (Dan Carter, Eytan Bardan); Department of

${ }^{\mathrm{b}}$ Gastroenterology, Rabin Medical Center, Petach Tikva (Ram Dickman); 'Sackler faculty of Medicine, Tel Aviv University, Tel Aviv (Dan Carter, Eytan Bardan, Ram Dickman), Israel

Conflict of Interest: None

Funding: The research was supported by a grant from Takeda Israel

Correspondence to: Dan Carter, MD, FEBGH, Department of Gastroenterology, Chaim Sheba Medical Center, $2^{\text {nd }}$ Sheba RD, Tel Hashomer, Israel, e-mail: dr.dancarter@gmail.com

Received 31 July 2017; accepted 21 October 2017; published online 16 November 2017

DOI: https://doi.org/10.20524/aog.2017.0214 of life, as well as substantial economical expenses $[5,6]$. General practitioners (GPs) are the main providers of medical management for chronic constipation in adults; in a survey conducted in the USA, only $14-18 \%$ of patients diagnosed with CC were evaluated by gastroenterology specialists (GIs) [7].

Most cases of CC are idiopathic, requiring treatment for symptom alleviation. The potential treatment possibilities for CC are many, including non-pharmaceutical approaches, such as changes in life habits or exercise [8], and pharmaceutical therapy consisting of fiber [9], osmotic laxatives such as polyethylene glycol [10], secretory laxatives (e.g., biascodyl [11], prokinetic medications (e.g., prucalopride [12] and secretagogues (e.g., lubiprostone) [13]. Although fiber and osmotic laxatives can usually be acquired without prescription, other types of medication can be prescribed only by physicians.

Although the possible treatment options for CC are abundant, effective treatment of CC remains challenging for both GPs and GIs. Poor correlation between symptoms and the underlying pathophysiology, the variety of symptoms regarded as $\mathrm{CC}$ and the heterogeneity of the patient population may explain the difficulty in tailoring the right treatment strategy for the individual patient. In the last years, a few national societies have released recommendations for the evaluation and treatment of CC [14-16]. However, in a recent study performed in five European countries, GPs still tended to diagnose CC by 
exclusion, whereas GI experts were more likely to diagnose CC on the basis of clinical judgment [17]. Furthermore, common approaches to the evaluation and treatment of CC among GIs and GPs had not been well defined and there were still unmet needs for an effective treatment of constipation.

Therefore, the aims of the current study were to evaluate the strategies and goals for the treatment of CC among GPs and GIs, and to examine the conceived limitations of the available treatment options.

\section{Materials and methods}

An internet questionnaire survey was conducted during January and February 2016 with the assistance of an internet research company (Tovanot, Inc., Kefar Saba, Israel), randomly targeting GIs and GPs. To qualify for the survey, GIs had to be board certified and GPs had to have been in practice for at least three years. A cover letter accompanying the questionnaire explained the purposes of the study.

The first part of the questionnaire covered the demographic and clinical characteristics, namely, age and sex of CC patients, the number of CC patients examined in a week, patients' main complaints and the major causes for referral for further GI consultations. The second part consisted of a structured questionnaire covering the central parameters for drug selection, the main goals of drug treatment, the definition of treatment efficacy, the perceived limitations of the available treatment, and questions regarding the treatment strategies and drug selection for CC.

\section{Statistical analysis}

A comparison of the responses of the GPs and GIs was conducted using SPSS for Windows (16.0; SSS Inc, Chicago, IL. USA). The chi-square or Fisher's exact tests were used for comparisons of dichotomous variables. A P-value $<0.05$ was reported as significant. All results are expressed as mean \pm standard deviation.

\section{Results}

Forty GIs and 132 GPs agreed to participate in the study and completed the internet survey. The maximal sample error ( $95 \%$ confidence level) was $\pm 13.4 \%$ and $\pm 8.8 \%$ among GIs and GPs, respectively. Both GIs and GPs evaluated an average of 11 patients with CC per week. Female patients were more commonly evaluated by both groups. GPs tended to treat older patients than GIs. Table 1 summarizes the main patient characteristics.

Thirty percent of the patients examined by the GPs were referred for further GI consultation. The main causes for referral were the presence of positive warning signs for significant gastrointestinal pathology, and the failure of therapy. Fig. 1 summarizes the major causes for referral for GI consultation.

The selection of drug treatment for CC differed between GIs and GPs. Table 2 summarizes the drug selection for CC by GPs and GIs. All GIs recommended osmotic laxatives as the first drug choice for CC, in comparison to only $70 \%$ of the GPs $(\mathrm{P}<0.001)$. Among GIs, polyethylene glycol (PEG) was the most common drug of choice, selected by $73 \%$ of all GIs, followed by lactulose (13\%). In case of failure of the first line of therapy, $53 \%$ of the GIs recommended secretory laxatives (mostly bisacodyl and senna), 25\% added fiber, 14\% changed to another type of osmotic laxative and $8 \%$ prescribed prucalopride. The third line of treatment among GIs consisted mainly of prucalopride (23\%), bisacodyl (20\%), paraffin oil (10\%), fleet enemas (16\%) and GoLYTELY ${ }^{\circledR}$ (PEG-3350 and electrolytes) (8\%). GPs chose PEG as first line of treatment in $41 \%$ of cases, lactulose in $29 \%$, bisacodyl in $13 \%$ and fiber in $10 \%$. The second line of treatment among GPs included secretory laxatives (32\%, mostly bisacodyl and senna), osmotic laxatives (31\%, mainly lactulose), and fiber supplementations (13\%). The drug choices for the third line of treatment were similar to the previous drug selection; secretory laxative were the most abundantly prescribed drugs (34\%), followed by osmotic laxatives in $16 \%$ of cases (mostly lactulose), glycerin suppositories in $11 \%$, fleet enema in $10 \%$, paraffin oil in $6 \%$. and prucalopride in $6 \%$.

Among GIs, the most significant parameters for drug selection included an established high safety profile (43\%), symptom improvement (30\%), and extensive clinical experience using the drug (20\%). Among GPs, the most noteworthy factors were symptom improvement (26\%), a high safety profile (25\%), and rapid onset of bowel movement (22\%) (Fig. 2).

The definition of drug efficacy differed slightly between GIs and GPs. Among GIs, the most significant parameters were symptom improvement $(68 \%)$ and the request for represcription of the same drug (25\%). Among GPs, symptom

Table 1 Patients' profile as reported by GPs and GIs

\begin{tabular}{lccc}
\hline Characteristics & $\begin{array}{c}\text { GPs } \\
(\mathrm{n}=132)\end{array}$ & $\begin{array}{c}\text { GIs } \\
(\mathrm{n}=40)\end{array}$ & P-value \\
\hline No. of patients $\backslash$ week & 11.2 & 11.3 & 1 \\
Patients' age (years) & $3 \%$ & $5 \%$ & 0.9 \\
$18-20$ & $4 \%$ & $15 \%$ & 0.03 \\
$21-40$ & $5 \%$ & $23 \%$ & 0.003 \\
$41-50$ & $37 \%$ & $30 \%$ & 0.6 \\
$51-65$ & $51 \%$ & $28 \%$ & 0.016 \\
$>65$ & $34 \%$ & $33 \%$ & 1 \\
Sex (male) & & & \\
Complains & $45 \%$ & $62 \%$ & 0.9 \\
Abdominal discomfort & $44 \%$ & $53 \%$ & 0.44 \\
Abdominal distension & $31 \%$ & $31 \%$ & 1 \\
\hline Abdominal pain & & & \\
\hline
\end{tabular}

GI, gastrointestinal specialist; GP, general practitioner 
Table 2 Drug treatment choices for chronic constipation among GIs and GPs

\begin{tabular}{|c|c|c|}
\hline Treatment choices & GIs (\%) & GPs (\%) \\
\hline \multicolumn{3}{|l|}{$1^{\text {st }}$ line of therapy } \\
\hline PEG & 73 & 41 \\
\hline Lactulose & 13 & 29 \\
\hline Other osmotic laxative & 14 & \\
\hline Fiber & & 10 \\
\hline Bisacodyl & & 13 \\
\hline Other & & 7 \\
\hline \multicolumn{3}{|l|}{$2^{\text {nd }}$ line of therapy } \\
\hline Bisacodyl & 35 & 25 \\
\hline Senna & 18 & 7 \\
\hline Fiber & 25 & 13 \\
\hline Another type of osmotic laxative & 14 & 31 \\
\hline Prucalopride & 8 & 2 \\
\hline Other & & 22 \\
\hline \multicolumn{3}{|l|}{$3^{\text {rd }}$ line of therapy } \\
\hline Prucalopride & 23 & 6 \\
\hline Bisacodyl & 20 & $29(+5$ senna $)$ \\
\hline Fleet Enema & 16 & 10 \\
\hline Paraffin oil & 10 & 6 \\
\hline GoLYTELY $^{\circledR}$ & 8 & \\
\hline Lactulose & 6 & 16 \\
\hline Glycerin suppositories & & 11 \\
\hline Other & 9 & 17 \\
\hline
\end{tabular}

PEG, polyethylene glycol; GI, gastrointestinal specialist; GP, general practitioner improvement was also the most significant parameter (50\%), followed by rapid onset of bowel movement (Fig. 3).

The main conceived limitations of drug therapy for CC among GIs and GPs included unwanted side effects of the drug (53\% and $48 \%$, respectively) and, in the case of treatment with novel drugs, a lack of clinical experience using the drug (47\% and $24 \%$, respectively) (Fig. 4).

\section{Discussion}

This survey revealed interesting findings that require addressing in the future for a potential improvement in the medical treatment of CC. We found significant differences in the choice of drug treatment for CC among GIs and GPs, especially for the more resistant cases, as well as major alterations in the perception of drug efficiency.

GIs and GPs treated a similar number of patients with CC. Female patients consisted of the majority of patients evaluated for CC. This finding is in agreement with previous reports [18]. A third of the patients were referred for further evaluation by GIs, usually due to positive warning signs and the failure to achieve treatment goals. Similar results were published in a survey of the management of chronic constipation by Italian GPs [19].

We found significant difference in the drug choice for CC between GIs and GPs. GIs tended to treat CC patients with systematic approach starting with osmotic laxatives, moving further to secretory laxatives, and in the case of resistant CC, prescribing prucalopride as the third line of treatment. This approach is in agreement with previous published guidelines $[14,15]$ that found it more effective. The fact that fiber was usually not prescribed could be related to the abundance of dietary fiber consumption from vegetables

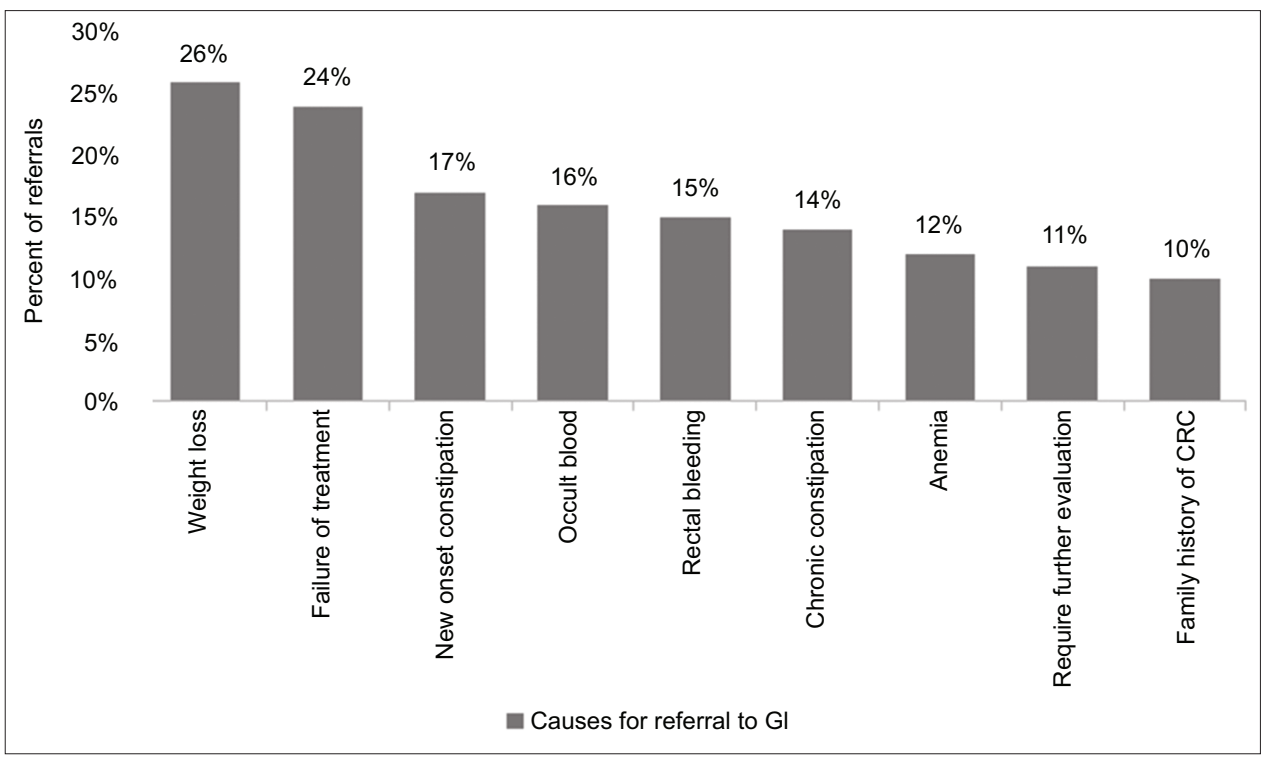

Figure 1 Major causes for referral for GI consultation GI, gastrointestinal specialist; CRC, colorectal cancer 


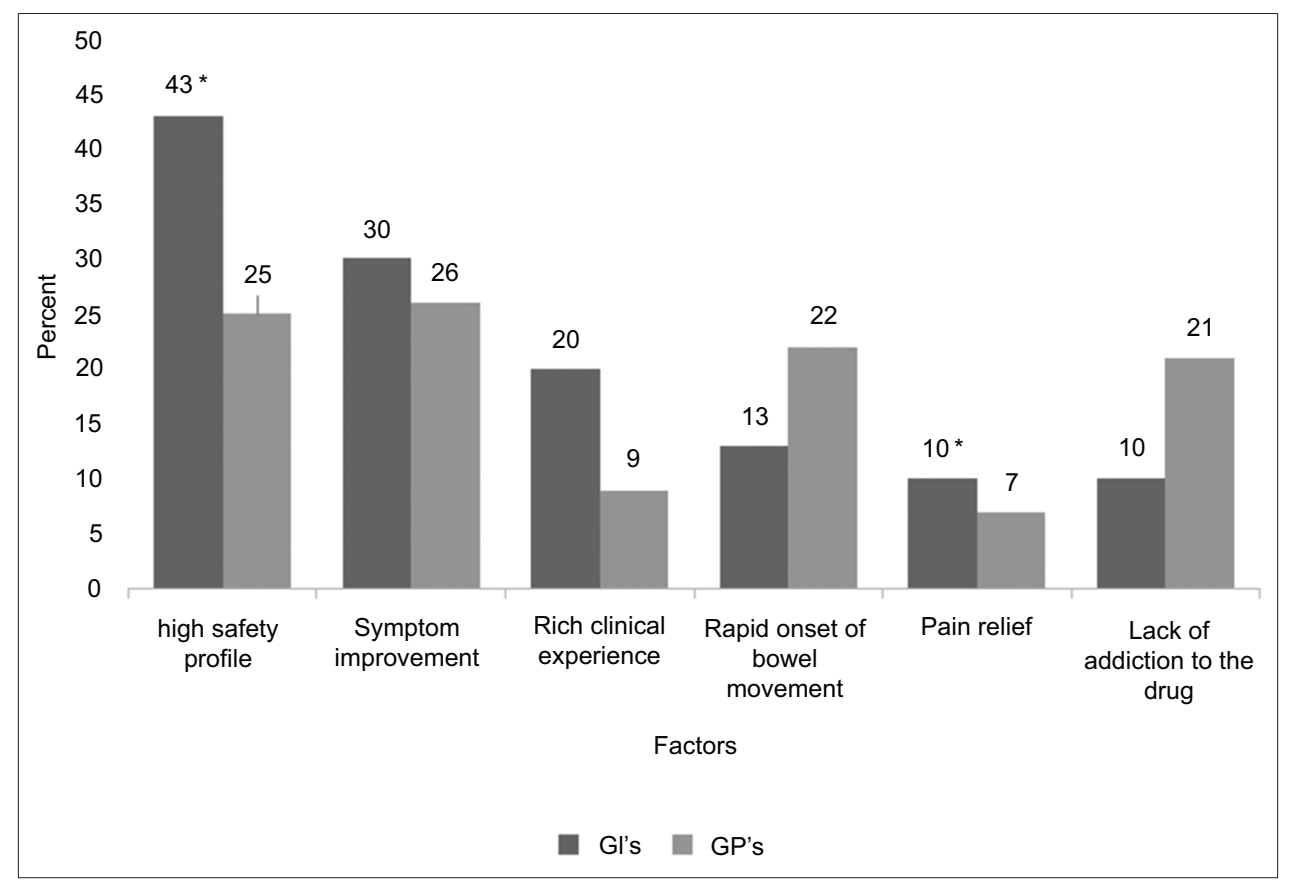

Figure 2 Significant parameters for drug selection for the treatment of chronic constipation among GIs and GPs, ${ }^{*} \mathrm{P}<0.05$ GI, gastrointestinal specialist; GP, general practitioner

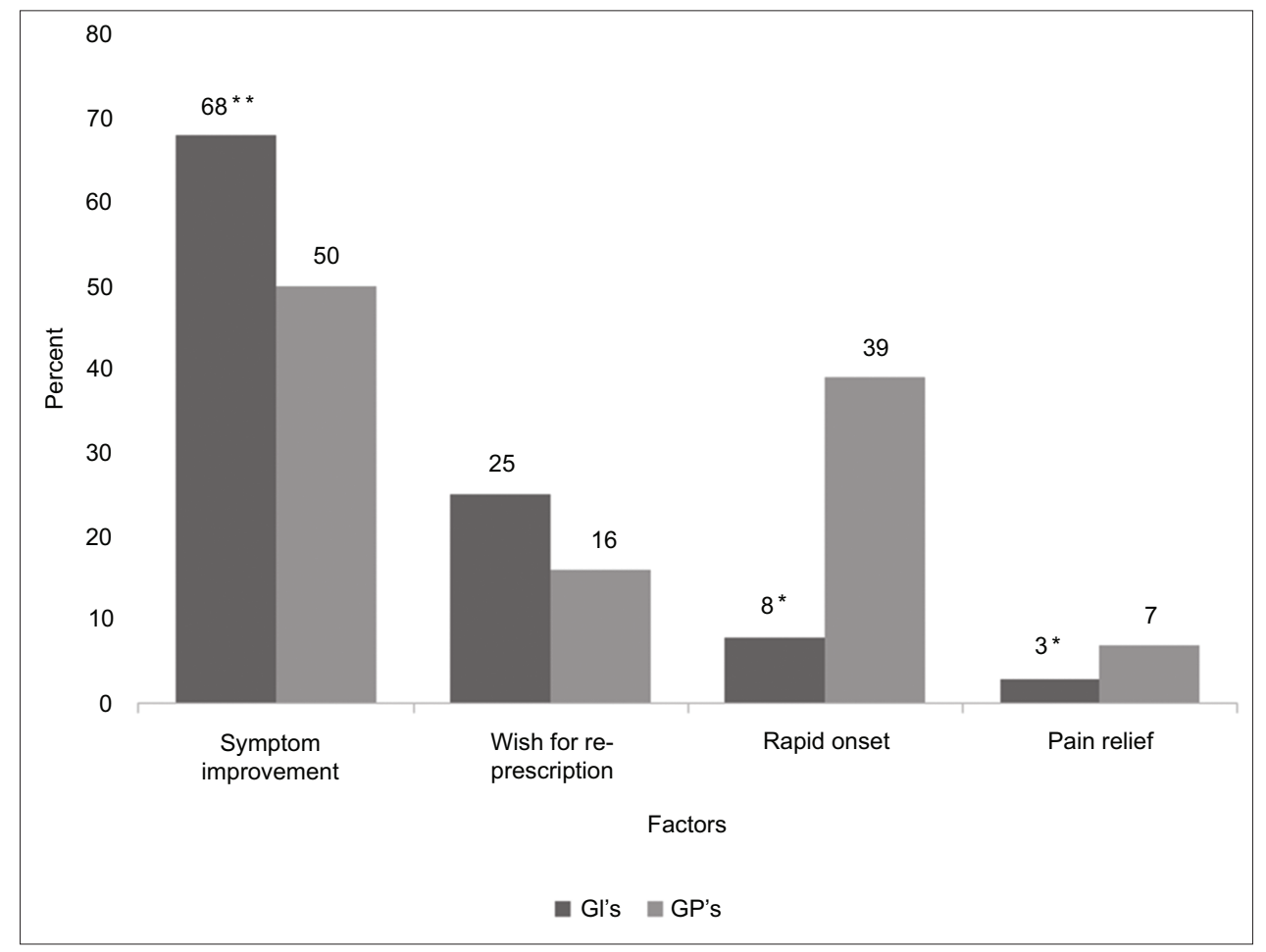

Figure 3 Main factors defining drug efficacy among GIs and GPs, ${ }^{\star} \mathrm{P}<0.05,{ }^{* *} \mathrm{P}<0.09$

GI, gastrointestinal specialist; GP, general practitioner

and fruits in the Israeli diet. On the other hand, the treatment strategy of the GPs toward CC was more heterogeneous, with prescription of osmotic and secretory laxatives as well as fiber as the first and second lines of therapy. Moreover, we found a gap in the ability to select efficient treatment for more resistant cases. These findings were addressed lately by publication of national guidelines toward the evaluation and treatment of CC for GPs and GIs [20]. We intend to revise the effectiveness of 


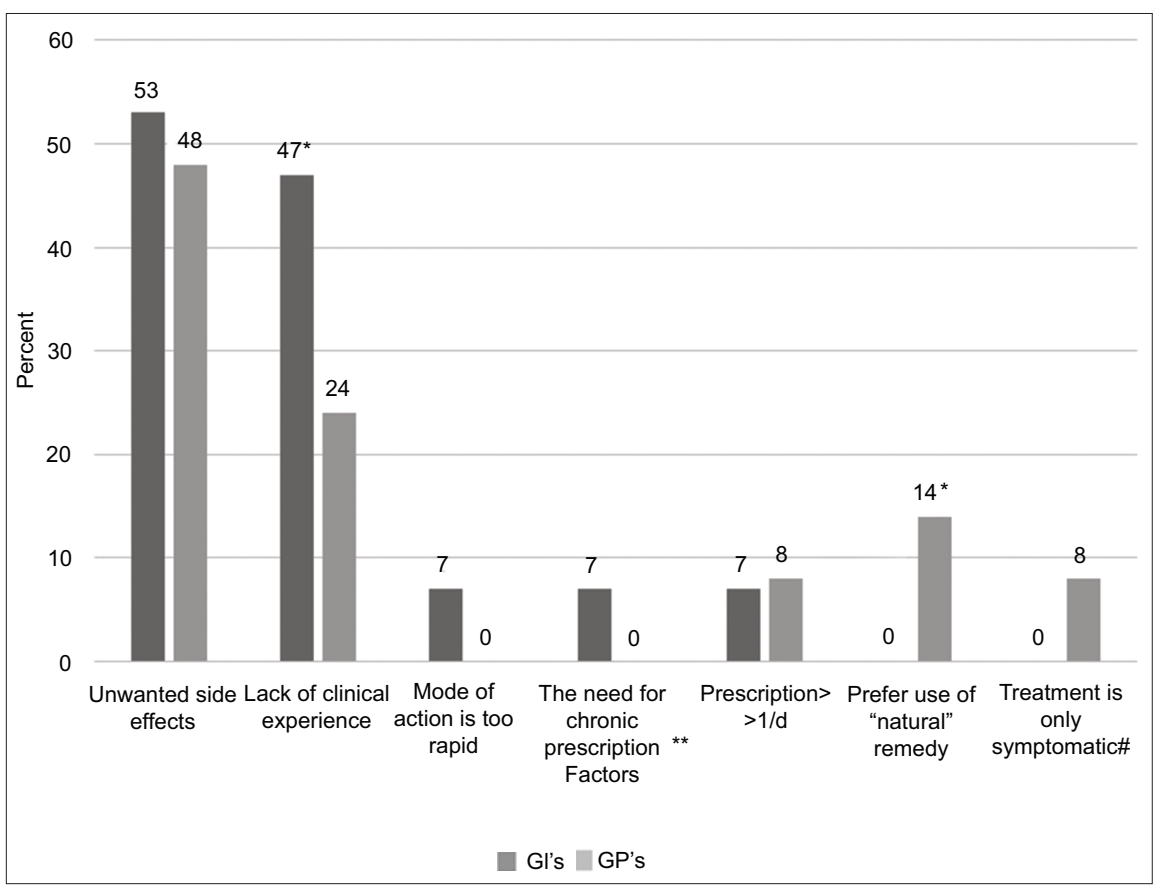

Figure 4 The conceived limitation of the drug therapy for chronic constipation among GIs and GPs, ${ }^{* *}$ The need of chronic prescription and not as an emergency treatment, \#Treatment is symptomatic and does not resolve the cause for constipation, ${ }^{*} \mathrm{P}<0.05$

GI, gastrointestinal specialist; GP, general practitioner

our guidelines in the next few years. A previous initiative for supporting primary care physicians by providing the primary care providers with education, decision-making support tools, and pre-referral management recommendations resulted in better treatment outcomes in the primary setting [21].

Interestingly, the perceived definition of drug efficacy for CC treatment was primarily related to the clinical benefit of the drugs, mainly symptom improvement, rapid onset of bowel movement and wish for re-prescription of the same drug both in GIs and GPs. However, the major parameters for the actual drug selection were primarily related to drug safety among GIs (drug safety and clinical experience), and, on the other hand, related to the clinical outcome among GPs (symptom improvement and rapid onset of bowel movement). These results may relate to the fact that GIs tend to prescribe newer medications, especially in case of failure of more "conservative treatment" with osmotic and secretory laxatives, extending the horizon of drug therapy for CC on one hand, but also increasing the chances for unwanted side effects with treatment options that might be considered to be less clinically practiced. The recent development of an extended range of new drugs for CC with which the GIs may be less familiar on one side, and the fact that CC is a benign condition necessitating treatment that is directed mainly towards symptom improvement on the other side, may also add to the reported requirement of established high safety profile of drug treatment for CC. This conclusion may also clarify the reasons for selecting unwanted side effects of the drugs and lack of clinical experience as the main conceived limitations of drug therapy for CC.

Our study has obvious limitations. The main limitation of the study is the possibility of a recall bias, typical for survey type studies.

\section{Summary Box}

\section{What is already known:}

- Chronic constipation (CC) affects $15 \%$ of the general western population

- The symptoms consistent with CC include straining, hard stools, sensation of incomplete evacuation, a sense of anorectal obstruction, manual maneuvers to facilitate defecation, and $<3$ spontaneous bowel movements per week

- The potential pharmaceutical treatment possibilities for CC include fiber, osmotic laxative, secretory laxatives, prokinetic medications, and secretagogues

\section{What the new findings are:}

- The selection of drug treatment for CC differed between GIs and GPs

- The most significant parameters for drug selection among GIs included high safety profile, symptom improvement, and a clinical experience using the drug. Among GPs, the most noteworthy factors were symptom improvement, high safety profile, and rapid onset of bowel movement

- The main conceived limitations of drug therapy for CC among GIs and GPs included unwanted side effects of the drug, and a lack of clinical experience in the case of treatment with novel drugs 
In conclusion, our results imply the need for better education of primary providers for efficient treatment of CC, as well as the need for a simple algorithm for the treatment of CC. Another important conclusion is the need for more large real-life population-based studies for the determination of the efficacy and safety of the emerging new drugs for CC.

\section{References}

1. Stewart WF, Liberman JN, Sandler RS, et al. Epidemiology of constipation (EPOC) study in the United States: relation of clinical subtypes to sociodemographic features. Am J Gastroenterol 1999;94:3530-3540.

2. Sonnenberg A, Koch TR. Physician visits in the United States for constipation: 1958 to 1986. Dig Dis Sci 1989;34:606-611.

3. Pare P, Ferrazzi S, Thompson WG, Irvine EJ, Rance L. An epidemiological survey of constipation in Canada: definitions, rates, demographics, and predictors of health care seeking. Am J Gastroenterol 2001;96:3130-3137.

4. Mearin F, Lacy BE, Chang L, et al. Bowel disorders. Gastroenterology 2016;150:1393-1407.e5.

5. Irvine EJ, Ferrazzi S, Pare P, Thompson WG, Rance L. Health-related quality of life in functional GI disorders: focus on constipation and resource utilization. Am J Gastroenterol 2002;97:1986-1993.

6. Wald A, Scarpignato C, Kamm MA, et al. The burden of constipation on quality of life: results of a multinational survey. Aliment Pharmacol Ther 2007;26:227-236.

7. Shah ND, Chitkara DK, Locke GR, Meek PD, Talley NJ. Ambulatory care for constipation in the United States, 1993-2004. Am J Gastroenterol 2008;103:1746-1753.

8. Rao SS, Rattanakovit K, Patcharatrakul T. Diagnosis and management of chronic constipation in adults. Nat Rev Gastroenterol Hepatol 2016;13:295-305.

9. Rao SS, Yu S, Fedewa A. Systematic review: dietary fibre and FODMAP-restricted diet in the management of constipation and irritable bowel syndrome. Aliment Pharmacol Ther 2015;41:1256-1270.

10. Dipalma JA, Cleveland MV, McGowan J, Herrera JL. A randomized, multicenter, placebo-controlled trial of polyethylene glycol laxative for chronic treatment of chronic constipation. Am J Gastroenterol 2007;102:1436-1441.

11. Kamm MA, Mueller-Lissner S, Wald A, Richter E, Swallow R, Gessner U. Oral bisacodyl is effective and well-tolerated in patients with chronic constipation. Clin Gastroenterol Hepatol 2011;9:577-583.

12. Sajid MS, Hebbar M, Baig MK, et al. Use of prucalopride for chronic constipation: a systematic review and meta-analysis of published randomized, controlled trials. J Neurogastroenterol Motil 2016;22:412-422.

13. Li F, Fu T, Tong WD, et al. Lubiprostone is effective in the treatment of chronic idiopathic constipation and irritable bowel syndrome: a systematic review and meta-analysis of randomized controlled trials. Mayo Clin Proc 2016;91:456-468.

14. Ford AC, Moayyedi P, Lacy BE, et al; Task Force on the Management of Functional Bowel Disorders. American College of Gastroenterology monograph on the management of irritable bowel syndrome and chronic idiopathic constipation. Am J Gastroenterol 2014;109(Suppl 1):S2-S26.

15. Tack J, Müller-Lissner S, Stanghellini V, et al. Diagnosis and treatment of chronic constipation-a European perspective. Neurogastroenterol Motil 2011;23:697-710.

16. Shin JE, Jung HK, Lee TH, et al. Guidelines for the diagnosis and treatment of chronic functional constipation in Korea, 2015 Revised Edition. J Neurogastroenterol Motil 2016;22:383-411.

17. Andresen V, Whorwell P, Fortea J, Auzière S. An exploration of the barriers to the confident diagnosis of irritable bowel syndrome: A survey among general practitioners, gastroenterologists and experts in five European countries. United European Gastroenterol J 2015;3:39-52.

18. Sanchez MI, Bercik P. Epidemiology and burden of chronic constipation. Can J Gastroenterol 2011;25 Suppl B:11B-15B.

19. Bellini M, Gambaccini D, Salvadori S, et al. Management of chronic constipation in general practice. Tech Coloproctol 2014; 18:543-549.

20. Carter D, Ron Y, Dickman R. The Israeli Neurogastroenterology Association recommendations for the evaluation and treatment of chronic constipation. Harefua [In Press].

21. Mallon D, Vernacchio L, Trudell E, et al. Shared care: a quality improvement initiative to optimize primary care management of constipation. Pediatrics 2015;135:1300-1307. 